\title{
A First Baseline for the Magnets in the High Luminosity LHC Insertion Regions
}

\author{
E. Todesco, H. Allain, G. Ambrosio, G. Arduini, F. Cerutti, R. De Maria, L. Esposito, S. Fartoukh, P. \\ Ferracin, H. Felice, R. Gupta, R. Kersevan, N. Mokhov, T. Nakamoto, I. Rakno, J. M. Rifflet, L. \\ Rossi, G. L. Sabbi, M. Segreti, F. Toral, Q. Xu, P. Wanderer, R. van Weelderen
}

\begin{abstract}
The High Luminosity LHC (HL-LHC) project aims at accumulating $3000 \mathrm{fb}^{-1}$ in the years 2023-2035, i.e. ten times more w.r.t. the nominal LHC performance expected for 20102021. One key element to reach this challenging performance is a new insertion region to reduce the beam size in the interaction point by approximately a factor two. This requires larger aperture magnets in the region spanning from the interaction point to the matching section quadrupoles. This aperture has been fixed to $150 \mathrm{~mm}$ for the inner triplet quadrupoles in 2012 . In this paper we give a first baseline of the interaction region. We discuss the main motivations that lead us to choose the technology, the combination of fields/gradients and lengths, the apertures, the quantity of superconductor, and the operational margin. Key elements are also the constraints given by the energy deposition in terms of heat load and radiation damage; we present the main features related to shielding and heat removal.
\end{abstract}

Index Terms - superconducting accelerator magnets, dipoles, quadrupoles, low-temperature superconductors

\section{INTRODUCTION}

$\mathrm{T}$ HE first proposal for increasing LHC luminosity goes back to the beginning of the century [1], relying on a more intense beam and on a smaller size of the beam at the interaction point. In order to reach this second target, one has to replace the 70 -mm-diameter inner triplet with a larger aperture magnet. First proposals focused on a $90-\mathrm{mm}$-aperture inner triplet quadrupole using the $\mathrm{Nb}_{3} \mathrm{Sn}$ technology. This motivated the LHC Accelerator Research Program (LARP) [2], launched in the US during the LHC construction. Successive studies showed that triplet apertures larger than $90 \mathrm{~mm}$ can bring additional performance, while shielding the magnets from the collision debris $[3,4]$.

In November 2011 a design study for the LHC luminosity upgrade has been launched, with the target of delivering a technical design for the Interaction Region (IR) layout by 2015 [5]. After the selection of the triplet aperture in July 2012, we have now taken the main choices for the other

Manuscript received July 14, 2013.

E. Todesco, H. Allain, G. Arduini, F. Cerutti, R. De Maria, L. Esposito, S. Fartoukh, P. Ferracin, R. Kersevan, L. Rossi, R. van Weelderen, are with CERN, 1211 Geneva 23, Switzerland. (e-mail: Ezio.Todesco@cern.ch)

G. Ambrosio, N. Mokhov, I. Rakno are with Fermilab, Batavia IL (USA).

H. Felice, G. L. Sabbi are with LBL, Berkeley CA (USA).

P. Wanderer, R. Gupta are with BNL, Upton NY (USA).

T. Nakamoto, Q. Xu are with KEK, Tsukuba (Japan).

M. Segreti, J. M. Rifflet are with CEA, Saclay (France).

F. Toral is with CIEMAT, Madrid (Spain). magnets of the IR (correctors, separation/recombination dipoles, ...), such as technology, fields, apertures and lengths (see Table 1). Here we summarize these choices, giving main motivations and possible alternatives.

\section{TRIPLET QXF}

The triplet is the source of performance: we selected the $\mathrm{Nb}_{3} \mathrm{Sn}$ technology giving larger peak field, i.e., larger apertures and/or larger gradients. The 70-mm, $200 \mathrm{~T} / \mathrm{m}$ of the LHC baseline will be replaced by a $150-\mathrm{mm}$ aperture triplet operating at $140 \mathrm{~T} / \mathrm{m}$ (see Table 1), allowing half the beam size and increased peak luminosity up to a factor four [6] . Thanks to $\mathrm{Nb}_{3} \mathrm{Sn}$, the increase in length is only $30 \%$ w.r.t. LHC, i.e., we go from a $30-\mathrm{m}$-long to a 40 -m-long triplet (see Fig. 1). The design of this magnet relies on 10 years of effort by the LARP collaboration [7]. The inner triplet quadrupole QXF [8] is a scaled-up version of the HQ quadrupole [7], successfully built in two 1-m-long models. In order to maximize the performance, a challenging margin of $\sim 20 \%$ on the loadline has also been chosen. The design and the status of the project is presented in [8]. The peak field is $12.1 \mathrm{~T}$.

TABLE I

PARAMETERS OF THE MAIN HL LHC MAGNETS

\begin{tabular}{|c|c|c|c|c|c|c|}
\hline & & $\begin{array}{c}\text { Triplet } \\
\text { Q1,Q3/Q2a,b }\end{array}$ & $\begin{array}{c}\text { Orbit } \\
\text { corrector }\end{array}$ & $\begin{array}{c}\text { Sep. } \\
\text { dipole } \\
\text { D1 } \\
\end{array}$ & $\begin{array}{c}\text { Recom. } \\
\text { dipole } \\
\text { D2 }\end{array}$ & $\begin{array}{c}\text { Large 2-in- } \\
1 \text { quad } \\
\text { Q4 } \\
\end{array}$ \\
\hline Aperture & $(\mathrm{mm})$ & 150 & 150 & 150 & 105 & 90 \\
\hline Field & (T) & & 2.1 & 5.2 & 3.5 & \\
\hline Gradient & $(\mathrm{T} / \mathrm{m})$ & 140 & & & & 120 \\
\hline Mag. Length & $(\mathrm{m})$ & $8.0 / 6.8$ & $1.2 / 2.2$ & 6.7 & 10.0 & 4.5 \\
\hline Int field & $(\mathrm{T} \mathrm{m})$ & & $2.5 / 4.5$ & 35 & 35 & \\
\hline Int gradient & (T) & $1120 / 938$ & & & & 544 \\
\hline Peak field & $(\mathrm{T})$ & 12.1 & 3.9 & 6.1 & 4.1 & 5.9 \\
\hline Current & $(\mathrm{kA})$ & 17.5 & 2.2 & 11.0 & 6.8 & 16.0 \\
\hline j overall & $\left(\mathrm{A} / \mathrm{mm}^{2}\right)$ & 528 & 455 & 1695 & 1040 & 2458 \\
\hline Loadline margin & $(\%)$ & $18 \%$ & $45 \%$ & $30 \%$ & $56 \%$ & $20 \%$ \\
\hline Stored energy & $(\mathrm{MJ} / \mathrm{m})$ & 1.440 & 0.090 & 0.294 & 0.140 & 0.204 \\
\hline Saturation & $(\%)$ & $9.0 \%$ & $0.0 \%$ & $9.0 \%$ & $13.0 \%$ & \\
\hline Material & & $\mathrm{Nb}_{3} \mathrm{Sn}$ & $\mathrm{Nb}-\mathrm{Ti}$ & $\mathrm{Nb}-\mathrm{Ti}$ & $\mathrm{Nb}-\mathrm{Ti}$ & $\mathrm{Nb}-\mathrm{Ti}$ \\
\hline No. layers & & 2 & $1+1$ & 1 & 1 & 1 \\
\hline Cable width & $(\mathrm{mm})$ & 18.1 & 4.37 & 15.1 & 15.1 & 15.1 \\
\hline Cable thick. in. & $(\mathrm{mm})$ & 1.405 & 0.819 & 1.362 & 1.362 & 1.362 \\
\hline Cable thick. ou. & $(\mathrm{mm})$ & 1.595 & 0.871 & 1.598 & 1.598 & 1.598 \\
\hline Ins. thick rad & $(\mathrm{mm})$ & 0.150 & 0.105 & 0.130 & 0.130 & 0.160 \\
\hline Ins. thick azi & $(\mathrm{mm})$ & 0.150 & 0.105 & 0.110 & 0.110 & 0.145 \\
\hline No. strands & & 40 & 18 & 36 & 36 & 36 \\
\hline Strand diam & $(\mathrm{mm})$ & 0.85 & 0.48 & 0.825 & 0.825 & 0.825 \\
\hline $\mathrm{Cu} / \mathrm{NonCu}$ & & 1.2 & 1.75 & 1.95 & 1.95 & 1.95 \\
\hline
\end{tabular}



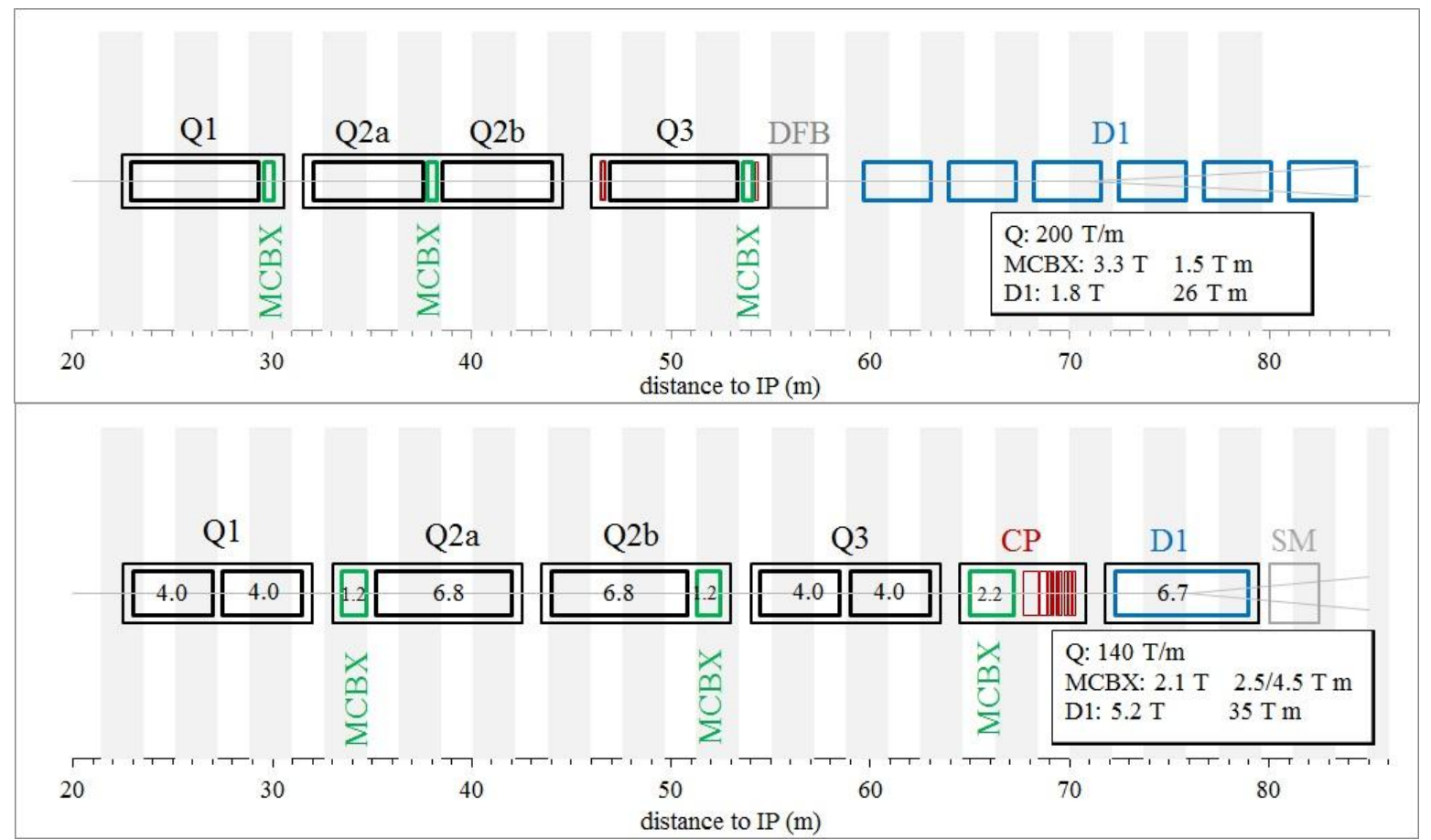

Fig. 1. Layout of the LHC (top), and HL-LHC (bottom) from the first quadrupole to the separation dipole. DFB in the top lay-out is the cryogenic feedbox hosting the current leads, while SM in the bottom lay-out is the shuffling module hosting the SC link coming from new DFB located on ground surface.

The structure (see Fig. 2) to manage the stress of $150 \mathrm{MPa}$ due to Lorentz force is given by an aluminum cylinder and loaded with bladders and keys, allowing precise stress control [9]. One of the main challenges of this magnet is the protection [10,11,12]: the time margin available to the protection system to quench the magnet is of the order of $40 \mathrm{~ms}$ (it is $100 \mathrm{~ms}$ in the LHC dipoles), i.e. just at the limit of present electronics and quench heaters.

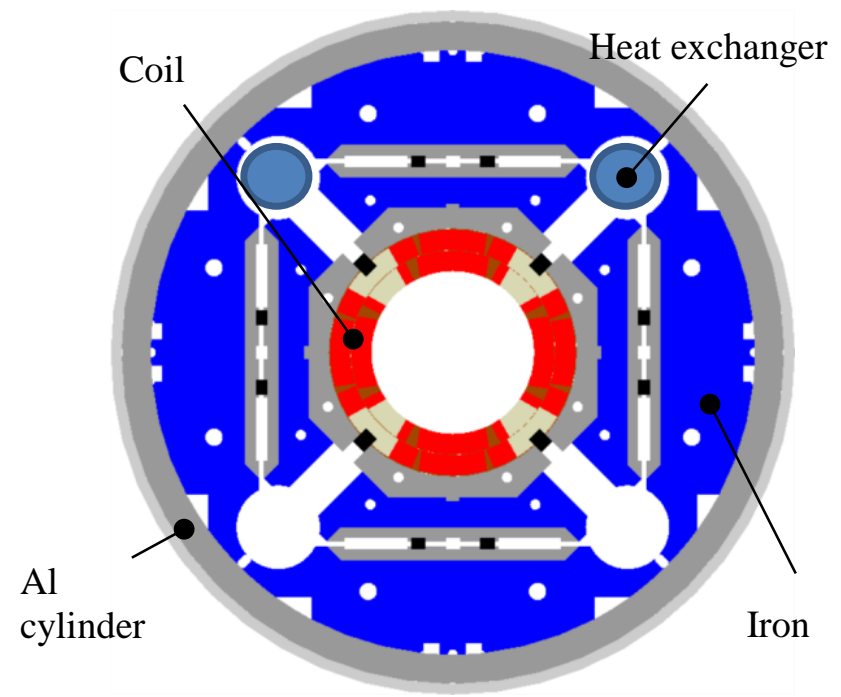

Fig. 2. Cross-section of the inner triplet [8].

\section{SEPARATION DIPOLE D1}

The longer space needed by the triplet is recovered by replacing the resistive D1 with a superconductive magnet (see Fig. 1). The separation dipole has the same aperture as the triplet, i.e. 150-mm-aperture, with an operational field of $5.2 \mathrm{~T}$ and a length of $6.7 \mathrm{~m} \mathrm{[13]}$. This magnet, as the triplet, operates in a regime of strong saturation (about 9\%, see Table 1). Considerable work has been carried out on the iron shape to optimize the field quality at high field. Fearing a large heat load, a margin of $30 \%$ on the loadline has been initially taken, and an aperture of $160 \mathrm{~mm}$ to allow for thicker shielding. The results of the energy deposition simulations [14] showed that the heat load is not significant with the heavy shielding, allowing reduction of the aperture to $150 \mathrm{~mm}$ (see Table 1). The mechanical structure is based on a precollaring, with prestress given by the iron and shell welding (see Fig. 3). The assembly prestress needed to keep the coil compressed at nominal current is $\sim 70 \mathrm{MPa}$. Details on the design are given in [13]. We are also considering the option of reducing the margin to 20\%: this would increase the field to $5.9 \mathrm{~T}$, and reduce the length to $6 \mathrm{~m}$, at the price of a higher saturation component. The drawback is a larger stress, which could reach the limits of insulation [13] during the assembly phases.

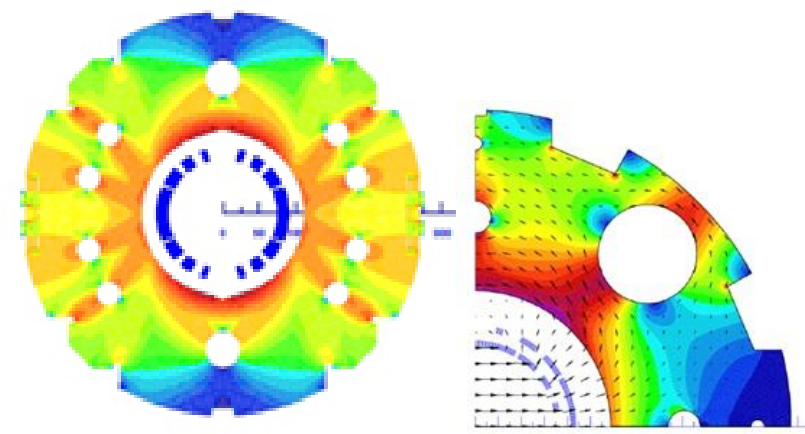

Fig. 3. Cross-section of the D1(left) [13] and tentative cross-section of the orbit corrector (right) [16].

The option of a $\mathrm{Nb}_{3} \mathrm{Sn}$ magnet, considered in the past, [15] has been now discarded, as the gain of a few meters (less than four, with an $11 \mathrm{~T}$ dipole) is not considered critical in this 
location and has no effect on performance. In addition, with such a large aperture the stresses would have been at the limit of the $\mathrm{Nb}_{3} \mathrm{Sn}$ damage level.

\section{ORBIT CORRECTORS}

Horizontal and vertical orbit correctors are needed at each quadrupole. The required bending strength is $2.5 \mathrm{~T} \cdot \mathrm{m}$ close to Q1 and Q2, and 4.5 T.m close to Q3. Given the large aperture, a cos theta option without nested coils, even with a large field as $4 \mathrm{~T}$, would need $0.5 \mathrm{~m}$ of straight part, plus $0.2 \mathrm{~m}$ of ends, plus space for connections [16]. Therefore one would need for the $2 \mathrm{~T} \cdot \mathrm{m}$ case at least $2 \mathrm{~m}$ per corrector (horizontal and vertical), with a non-negligible impact on performance. With a nested option and a moderate field of $2.1 \mathrm{~T}$, we manage to have a 1.2-m-long magnet (see Fig. 1).

The global saving on the whole triplet length given by the nested layout is more than $3 \mathrm{~m}$, equivalent to a $10 \%$ increase of the triplet gradient. Preliminary studies have been carried out, with the development of an 18-strand cable of very small width $(4.5 \mathrm{~mm}$, see Table 1 and Fig. 3) [16]. With a one-layer coil, $2.1 \mathrm{~T}$ operational field is reached with a very comfortable $45 \%$ margin on the loadline (see Table 1). The main challenge of this magnet is a mechanical design that manages the torque of $90 \mathrm{kN} \cdot \mathrm{m} / \mathrm{m}$ when both correctors are at nominal field.

The option of increasing the field to $3 \mathrm{~T}$, thus reducing the margin to $30 \%$, would gain $1-1.5 \mathrm{~m}$ at the price of twice the torque.

\section{HIGH ORDER MAGNET CORRECTORS}

Today, we have nested correctors providing a very compact layout (see Fig. 1, top). In the HL-LHC era, the sensitivity of the beam to high order multipoles in the triplet will be large and for several multipoles there are no beam observables. The requirement is to have nine high order correctors, starting with a skew quadrupole, and going up to order 6 . In order to have a transparent operation in this critical region of the accelerator, a non-nested option has been considered, which also avoids cross-talk between different magnets.

We considered the superferric option developed in CIEMAT [17] for the EU program FP7-SLHC. Even though the field is limited by the iron saturation, this option has the appealing advantage of allowing very short ends, as the windings are rectangular racetracks (see Fig. 4).
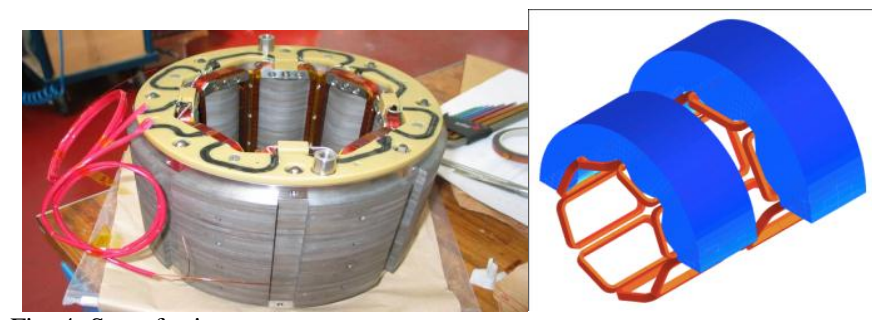

Fig. 4. Superferric correctors.

$\mathrm{MgB}_{2}$ [18] or Nb-Ti conductors are considered, with the iron at $1.9 \mathrm{~K}$. The short end allows a compact layout even in the case of a non-nested geometry. The whole block of correctors takes a couple of meters, plus the skew quadrupole, which is $0.7 \mathrm{~m}$ long. Most of the objects are $\sim 0.1 \mathrm{~m}$ long.
Prototypes have been already been built (with $140 \mathrm{~mm}$ aperture) in the framework of the Phase I upgrade [17].

\section{HEAT LOAD AND RADIATION DAMAGE FROM Q1 to D1}

The larger peak and integrated luminosities pose issues on the heat load and on the radiation damage, respectively. The solution is to have a large aperture triplet, to make space for thick shielding: results from energy deposition simulations $[14,19,20]$ show that the magnets can be protected from the collision debris through appropriate shielding. This is an additional reason (beyond getting to smaller $\beta^{*}$ ) for requiring larger triplet apertures.

The mechanical requirement is to have a 4-mm-thick stainless steel cold bore to withstand pressure during a quench, plus a $2-\mathrm{mm}$-thick beam screen, internally coated with $\mathrm{Cu}$ to decrease the impedance. In addition, we add a $6 \mathrm{~mm}$ thick absorber in the angles where the load is larger, using high-Z, high density material (tungsten). Moreover, $16 \mathrm{~mm}$ tungsten absorbers have been considered in the Q1, which has ends exposed to considerable debris radiation and, on the other hand, does not suffer from stringent aperture limitation (see Fig. 5). With this shielding one can operate in HL-LHC at the same levels of LHC, i.e. below $2 \mathrm{~mW} / \mathrm{cm}^{3}$ peak heat load, and $25 \mathrm{MGy}$ of radiation dose (see Fig. 6) despite the five times larger peak luminosity, and the ten times larger accumulated debris from the interaction point.
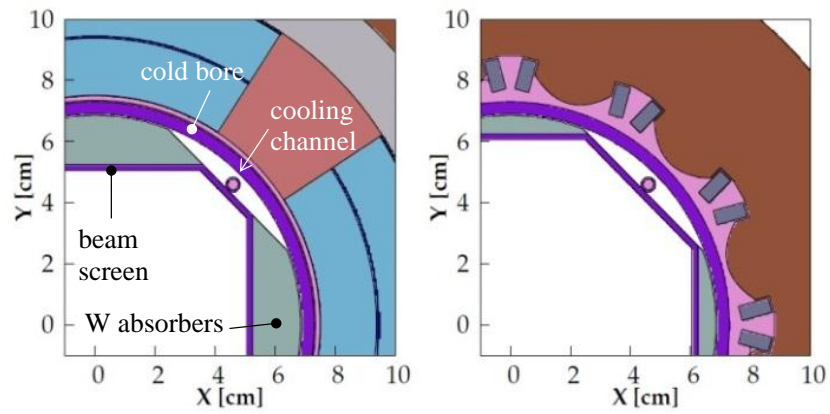

Fig. 5. Cold bore, beam screen and W shielding in the triplet and correctors.

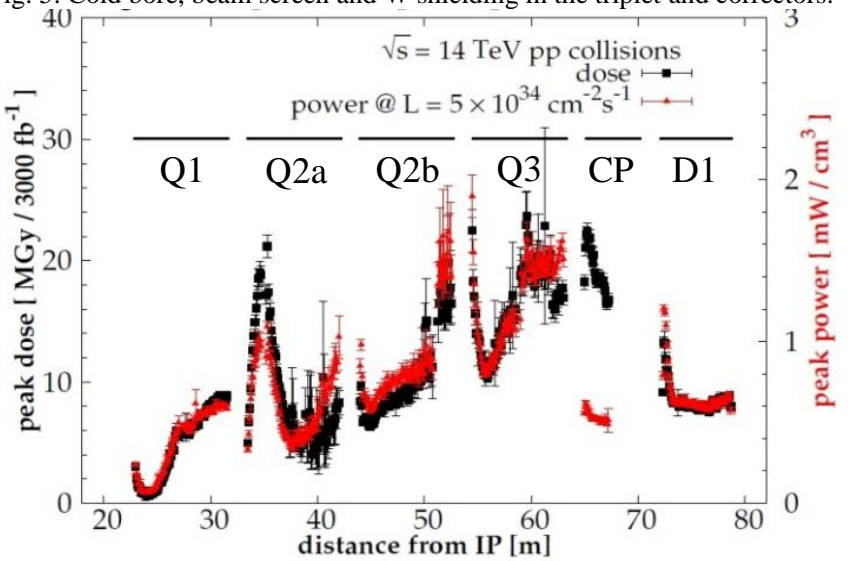

Fig. 6. Peak heat load and peak radiation dose for HL LHC in the coil (peak luminosity of $5 \times 10^{34} \mathrm{~cm}^{-2} \mathrm{~s}^{-1}$ and $3000 \mathrm{fb}^{-1}$ of integrated luminosity).

\section{CoOling}

The heat load coming from the collision debris is the dominating factor of the cooling system. In the HL-LHC the particles coming from the interaction point, bent by the 
magnetic field of the triplet, correctors and separation dipole, deposit about $1300 \mathrm{~W}$ on the string from Q1 to D1 shown in Fig. 1. Half of this is intercepted by the beam screen, which has an operational temperature of 5-20 K, and half goes on the so-called cold mass, i.e. the part of the magnet at $1.9 \mathrm{~K}$. The removal of the heat load at $1.9 \mathrm{~K}$ is done through two separate systems of heat exchangers, one covering the triplet and the 1.2-m-long orbit correctors, and the second one covering D1 and the corrector package ( $\mathrm{CP}$, see Fig. 1 bottom). For the triplet, the 77-mm-diameter heat exchangers are at $45^{\circ}$, respecting the quadrupole symmetry (see Fig. 2). Two out of the four holes are used by heat exchangers. The same holes are present in the $2 \mathrm{~m}$ long orbit correctors. On the other hand, the separation dipole and the high order correctors are cooled by one $50 \mathrm{~mm}$ diameter heat exchanger, at $90^{\circ}$ (see Fig. 3).

The beam screen receives $700 \mathrm{~W}$ from the debris, plus another $100 \mathrm{~W}$ from the circulating beam (electron cloud, impedance, plus other effects). The cooling needs two tubes of $\sim 8 \mathrm{~mm}$ diameter. The engineering of this component is a critical part of the project.

\section{RECOMBINATION DIPOLE D2}

This magnet is placed at 138-148 $\mathrm{m}$ from the IP (see Fig. 2, compared to the $72-78 \mathrm{~m}$ position of $\mathrm{D} 1$ readable on the horizontal axis), where the D1 kick has separated the beams to the nominal distance. It has to provide $35 \mathrm{~T} \mathrm{~m}$ of integrated field to bring the beams back on parallel paths. The main challenges come from the impact of the two-in-one magnetic structure on field quality [21]. Apertures are increased from the present baseline of $80 \mathrm{~mm}$ to $95-105 \mathrm{~mm}$. The nominal field is $3.5 \mathrm{~T}$, with a $10 \mathrm{~m}$ length, and a very comfortable $56 \%$ margin (see Table 1). The larger aperture, the beam separation fixed, has the drawback of creating a magnetic coupling between the two apertures (see Fig. 7), producing very large systematics $b_{2}$ (70 units) and $b_{4}$ (5 units). Moreover, the saturation produces a large $b_{3}$ (50 units) and $b_{5}$ (20 units). A careful shaping of the iron, as done in RHIC or in the LHC dipole, is needed to reduce these components. The result of this optimization will determine the final aperture and level of operational field.

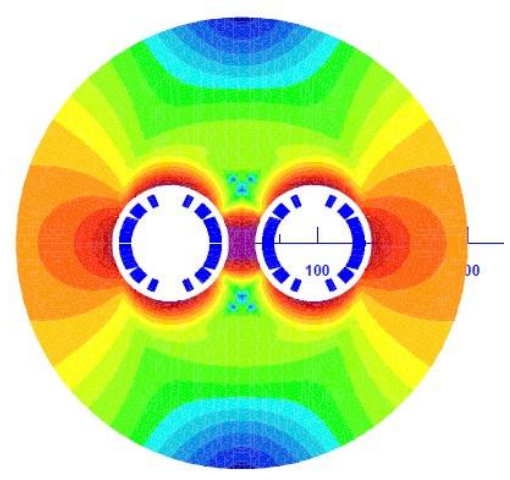

Fig. 7. Tentative cross-section of recombination dipole D2.

One can also consider the option of a larger field of $5 \mathrm{~T}$ to get a $7 \mathrm{~m}$ long magnet, thus saving $3 \mathrm{~m}$ of space, which in this region is more important than, for example, near D1, because of the presence of the new crab cavities [22]. The drawback is a smaller but still comfortable margin, at the expense of larger saturation, possibly making field quality considerably worse.

\section{TWO-IN-ONE LARGE APERTURE QUADRUPOLE Q4}

Q4 is the first quadrupole with beam circulating in separated vacuum chambers and is placed after the recombination dipole $\mathrm{D} 2$, at $168 \mathrm{~m}$ from the IP. Its aperture is increased from the present value of $70 \mathrm{~mm}$ to $90 \mathrm{~mm}$. As for the D2 case, there is a large magnetic coupling between the apertures, because of the larger aperture and the beam separation fixed to $194 \mathrm{~mm}$ [23]. For this reason a pretty thin coil of $15 \mathrm{~mm}$ width, using one layer of LHC main dipole cable, has been adopted. With a margin of $20 \%$ on the loadline one reaches $120 \mathrm{~T} / \mathrm{m}$, still providing a short magnet of $4.5 \mathrm{~m}$ (see Fig. 8). Larger cable width would leave no space for the iron.

The mechanical structure is based on self-supporting collars. The design team adopted the enhanced insulation [24] tested in the MQXC model [25] as baseline. This allows helium to reach the strands, giving a more efficient heat removal. An alternative design based on two layers of a thinner (7-mm-width) cable has been considered, which lowers operational current from 16 to $5 \mathrm{kA}$. Indeed, the one layer option requires no quench heaters, and can be done with pieces of LHC main cable production refused for being too short; therefore this design has been adopted.

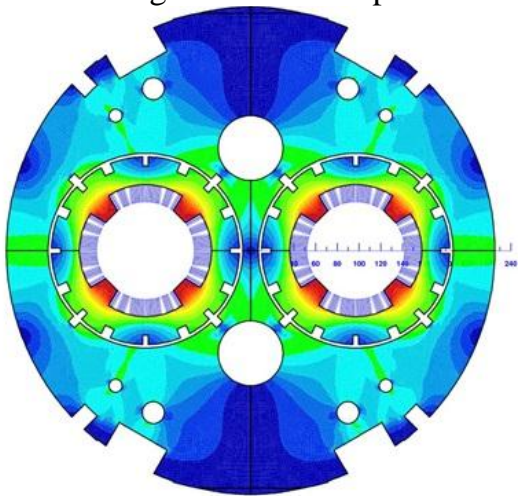

Fig. 8. Cross-section of large aperture quadrupole Q4.

\section{CONClusion}

The luminosity upgrade project HL LHC started in October 2010. After the selection of the triplet aperture of $150 \mathrm{~mm}$, done in July 2012, in the past year we focused on the definition of the baseline of the LHC interaction region.

In this paper we present the main choices for the technology, aperture, margin, operational field and gradients, lengths, margins and cables for the magnets from the triplet up to the fourth quadrupole $\mathrm{Q} 4$, in the region where the beams are separated and travel in two different apertures. Alternative options are also discussed. We now plan to start the engineering work to be able to test the first short models in 2015-2016 to allow sufficient time for eventual modifications and then proceed to construction to meet the goal of installation in 2022.

\section{REFERENCES}

[1] O. Bruning, et. al, "LHC luminosity upgrade: a feasibility study", $L H C$ Project Report 626, 2002. 
[2] J. Strait, et. al, "Towards a new LHC interaction region design for a luminosity upgrade", Particle Accelerator Conference, pp. 42-42, 2003.

[3] J. P. Koutchouk, et al., "A concept for the LHC luminosity upgrade based on strong beta* reduction combined with a minimized geometric luminosity reduction factor", Particle Accelerator Conference, pp. 33873389, 2007.

[4] E. Todesco, et al., "Scaling laws for $\beta^{*}$ in the LHC interaction region", CERN 2007-002, pp. 61-80, 2007.

[5] L. Rossi, et al., "High Luminosity Large Hadron Collider: A description for the European Strategy Preparatory group", CERN-ATS-2012-236, 2012.

[6] S. Fartoukh, "Breaching the Phase I optics limitations for the HL-LHC", CERN-sLHC-Project Report 0053, 2011, also S. Fartoukh, "An achromatic telescopic squeezing scheme for the LHC upgrade", International Particle Accelerator Conference pp. 2088-90, 2011.

[7] P. Ferracin, "LARP $\mathrm{Nb}_{3} \mathrm{Sn}$ quadrupole magnets for the LHC luminosity upgrade", Adv. Cryo. Eng., vol. 55, pp. 1291, 2010.

[8] P. Ferracin, "Magnet design of the $150 \mathrm{~mm}$ aperture low- $\beta$ quadrupoles for the high luminosity LHC", IEEE Trans. Appl. Supercond. $\mathbf{2 4}$ (submitted).

[9] S. Caspi., "A new support structure for high field magnets", IEEE Trans. Appl. Supercond. vol. 12 pp 47-50, 2002.

[10] G. Manfreda, "Quench protection study of the $\mathrm{Nb}_{3} \mathrm{Sn}$ low- $\beta$ quadrupole for the LHC luminosity upgrade", IEEE Trans. Appl. Supercond. 24 (submitted).

[11] T. Salmi, "Protection heater delay in high field $\mathrm{Nb}_{3} \mathrm{Sn}$ accelerator magnets", IEEE Trans. Appl. Supercond. 24 (submitted).

[12] E. Todesco, "Quench limits in the next generation of magnets", in: Proceedings of WAMSDO workshop, CERN yellow report, to be published.
[13] Q. Xu, "Design of the separation dipoles for HL-LHC", IEEE Trans. Appl. Supercond. 24 (submitted).

[14] L.S. Esposito et al. "FLUKA energy deposition studies for the HLLHC", International Particle Accelerator Conference, pp. 1379-1341, 2013.

[15] A. den Ouden, et al., "Progress in the development of an 88-mm-bore $10 \mathrm{~T} \mathrm{Nb}_{3}$ Sn dipole magnet", IEEE Trans. Appl. Supercond., vol. 11, pp. 2668-2671, 2001.

[16] M. Karpinnen, talk given at LARP HI-Lumi meeting in Frascati, 2012 https://indico.cern.ch/conferenceDisplay.py?confId $=183635$

[17] F. Toral, "Development of superconducting corrector magnets with hard radiation resistance for LHC upgrade", IEEE Trans. Appl. Supercond. vol. 23, pp. 4101204, 2013.

[18] G. Volpini, private communication.

[19] G. Battistoni et al., "The FLUKA code: Description and benchmarking," AIP Conf. Proc., vol. 896, pp. 31, 2007.

[20] A. Ferrari et al., "FLUKA: A multi-particle transport code (Program version 2005)," CERN-2005-010.

[21] R. Gupta, talk given at LARP HI-Lumi meeting in Napa Valley, 2013 https://indico.fnal.gov/conferenceDisplay.py?confId=6164

[22] R. Calaga, et al., "A quarter wave design for crab crossing in the LHC", International Particle Accelerator Conference, pp. 121-123, 2012.

[23] M. Segreti, J. M. Rifflet, "Studies on large aperture quadrupoles - I, II and III report", http://www.cern.ch/hilumi/wp3

[24] M .La China, D. Tommasini, "Cable insulation scheme to improve heat transfer to superfluid helium in $\mathrm{Nb}-\mathrm{Ti}$ accelerator magnets", IEEE Trans. Appl. Supercond., vol. 18 pp. 1285-1288, 2008.

[25] G. Kirby, et al., "LHC IR upgrade Nb-Ti, $120 \mathrm{~mm}$ aperture model quadrupole, test results at $1.8 \mathrm{~K}$ ", IEEE Trans. Appl. Supercond. 24 (submitted). 